XL.

Aus dem pathologischen Institut der med. Akademie zu Osaka, Japan.

\title{
Ueber die toxischen und hämolytischen Wirkungen der Organautolysate.
}

\author{
Von \\ Y. Fukuhara, \\ Abtheilungsvorsteher des Instituts.
}

Im Organismus findet sich in grosser Verbreitung ein intracelluläres, von Salkowski (1) entdeckles Ferment, welches in Stande ist, Eiweiss in diffusible Spaltungsproducte umzuwandeln. Für diesen Fermentprocess hat Jacobi $(2,3)$ den Namen "Autolyse" vorgeschlagen. Es kann also nicht wundernehmen, dass die Autolyse in allen Organen gefunden ist. So fand Jacobi (3) Autolyse in den Jungen, Hedin und Rowland (4) Proteolyse in dem Presssaft der Milz, der Lymphdrüsen und der Nieren. Weiterhin constatirte Okerblom (5) Autolyse in den Nebennieren, Matthes (6) in der Placenta, Kutscher (7) in der Thymusdrüse, Kutscher und Seemann (8) in der Darmwand, Langstein und Neabauer (9) im Uterus, Reh (10) in den Lymphdrüsen. Unter Autolyse verstehen wir heutzutage die Summe der chemischen Vorgänge, die sich in thierischen Organen oder Flüssigkeiten abspiclen, wenn wir dieselben nach der Abtrennung vom Gesammtorganismus unter den Bedingungen aufbewahren, welche die Mitwirkung von Mikroorganismen sowohl durch die Anwendung antiseptisch wirkender Substanzen als auch durch das Verfahren der Asepsis, ausschliessen. Nun ist es selbstverständlich, dass solche autolytische, schon bei Lebzeiten im Organismus vor sich gehende Processe - z. B. die Colliquationsnekrose, die Resorption des pneumonischen Exsudats u. A. m. - nicht nur anatomische Veränderungen nach sich ziehen können, sondern dass auch die Resorption ler autoJytischen Producte einen Einfluss auf den Organismus auszuüben vermag. Früher hat bereits Petry (11) darauf aufmerksam gemacht, dass im Carcinomgewebe der Autolyse entsprechende, chemische Unwandlungen eintreten, dass anch proteolylische Enzyme augenscheinlich in sehr erheblicher Menge darin enthalten sind, wie sie sonst in keinem Organ vorhanden sind. Er hat auch damit eine Reihe Lrscheinungen in Be- 
ziehung gebracht, welche man bei Individuen mit malignen Geschwülsten beobachten kann.

Meine Untersuchungen zerfallen in folgende:

1. Untersuchung der hämolytischen Wirkung des Autolysats.

2. Untersuchung der Giftigkeit des Autolysats.

\section{Die hämolytischen Wirkungen des 0rganautolysats.}

Die Beobachtungen über die hämolytische Wirkung von Organextracten sind von Metschnikoff (12), Schibajama (13ł, Klein (14), Tarasseviteh (15) und von Korschun und Morgenroth (16), sowie von Hagitani (17) aus unserem Laboratorium unter Director Prof. Sata's Leitung erschienen. Was die hämolytischen Wirkungen des Organautolysats betrifft, wurden sie bisher noch nicht genau untersucht.

In meiner Untersuchung werden dic Autolysate in folgender Weise hergestellt. Alle frisch aufgenommenen Organe wurden fein zerhackt und nach Salkowski mit der zweifachen Menge Wasser nach Zusatz des üblichen Ueberschusses von Toluol und Chloroform bei $38^{\circ} \mathrm{C}$. im Brutschrank unter häufigem Umschütteln mehrere Tage hindurch digerirt, im Vacuum freies Ammoniak ausgetrieben und durch Tonkerze filtrirt. Zur Untersuchung der Hämolyse wurden die Blutkörperchen verwendet, die vom Serum durch Centrifugiren möglichst befreit waren. Die Versuchsreihen wurden 2-3 Stunden im Thermostat bei $37^{\circ}$ und 10 Stunden im Eisschrank aufbewahrt und erst dann das Resultat festgestellt.

Das Autolysat von Rinderleber, -Milz, -Niere, sowie von Meerschweinchenleber, -Milz, -Niere und -Lunge wurde hinsichtlich der Wirkung auf das Blut der eigenen Species und der fremden geprüft.

' a b e $11 \mathrm{e} 1$.

Autolysat von Rinderleber (45 Tage lang autolysirt).

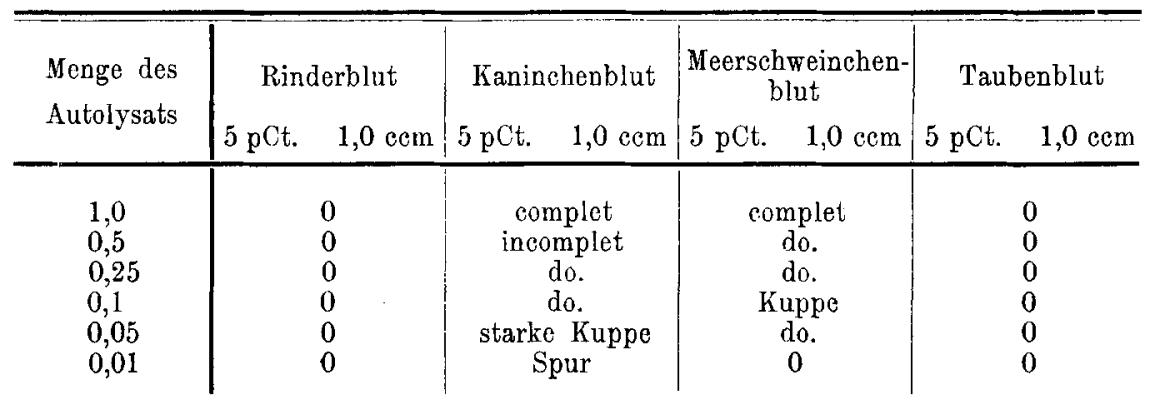

Autolysat von Rinderniere (35 Tage lang autolysirt).

\begin{tabular}{l|l|c}
1,0 & 0 & spurweise \\
0,5 & 0 & Kuppe \\
0,25 & 0 & do. \\
0,1 & 0 & do. \\
0,05 & 0 & do. \\
0,01 & 0 & do.
\end{tabular}


Autolysat von Rindermilz (\$5 Tage lang autolysirt).

\begin{tabular}{|c|c|c|c|c|c|c|c|c|}
\hline \multirow{2}{*}{$\begin{array}{l}\text { Menge des } \\
\text { Autolysats }\end{array}$} & \multirow{2}{*}{\multicolumn{2}{|c|}{$\begin{array}{l}\text { Rinderblut } \\
5 \text { pCt. } \quad 1,0 \mathrm{ecm}\end{array}$}} & \multicolumn{2}{|c|}{ Kaninchenblut } & \multicolumn{2}{|c|}{$\begin{array}{l}\text { Meerschweinchen- } \\
\text { blut }\end{array}$} & \multicolumn{2}{|c|}{ Taubenblut } \\
\hline & & & $5 \mathrm{pct.}$ & $1,0 \mathrm{~cm}$ & $5 \mathrm{pCt}$. & $1,0 \mathrm{ccm}$ & $5 \mathrm{pCt}$. & $1,0 \mathrm{cem}$ \\
\hline 1,0 & & 0 & starke & Kuppe & & 0 & & 0 \\
\hline 0,5 & & 0 & & do. & & 0 & & 0 \\
\hline 0,25 & & 0 & & uppe & & 0 & & 0 \\
\hline 0,1 & & 0 & & do. & & 0 & & 0 \\
\hline 0,05 & & 0 & & do. & & 0 & & 0 \\
\hline 0,01 & & 0 & & 0 & & 0 & & 0 \\
\hline
\end{tabular}

Autolysat von Meerschweinchenleber (37 Tage lang autolysirt).

\begin{tabular}{l|l|c|c|c}
1,0 & 0 & starke Kuppe & complet & 0 \\
0,5 & 0 & do. & do. & 0 \\
0,25 & 0 & Kuppe & do. & 0 \\
0,1 & 0 & do. & Kuppe & 0 \\
0,05 & 0 & 0 & 0 & 0 \\
0,01 & 0 & 0 & 0 & 0
\end{tabular}

Autolysat von Meerschweinchenniere (35 Tage lang autolysirt).

\begin{tabular}{l|l|c|c|c}
1,0 & 0 & - & - & 0 \\
0,5 & 0 & - & Kuppe & 0 \\
0,25 & 0 & incomplet & - & 0 \\
0,1 & 0 & starke Kuppe & - & 0 \\
0,05 & 0 & do. & - & 0 \\
0,01 & 0 & Kuppe & - & 0
\end{tabular}

Autolysat vou Meerschweinchenherz (35 Tage lang autolysirt).

\begin{tabular}{l|l|c|c|c}
1,0 & 0 & starke Kuppe & complet & starke Kuppe \\
0,5 & 0 & do & starke Kuppe & 0 \\
0,25 & 0 & 0 & 0 & 0 \\
0,1 & 0 & 0 & 0 & 0 \\
0,05 & 0 & 0 & 0 & 0 \\
0,01 & 0 & 0 & 0 & 0
\end{tabular}

Ls ist aus diesen Versuchen zu ersehen, dass die Empfänglichkeit des Blutes gegen das eigene Organautolysat verschieden ist, wie die einer fremden Blutart. Ob alle diese Autolysate das Blut des Individuums selbst lösen, konnte ich natürlich nicht untersuchen, halte es aber für wahrscheinlich. Ich musste also meine Untersuchung vor allem anstellen, um festzustellen, ob sich die hämolytischen Organautolysate durch den Nachweis von Complement und Amboceptor charakterisiren liessen. Dieser erste Zweifel veranlasste mich zunächst, die hämolytischen Autolysate in Bezug auf diejenigen Hanptcharacteristica zu untersuchen, die man bcim Studium der complexen Hämolysine kennen gelernt hat. Ich beginne mit den Versuchen, durch die das Verhalten des Organautolysats gegenüber höheren Temperaturen geprüft wird.

Tabelle 2.

Einwirkung erhitzter Organautolysate auf Kaninehenblut ( $1,0 \mathrm{cem} 5 \mathrm{pCt}$.). a) Autolysat von Rindermilz (45 Tage lang autolysirt).

\begin{tabular}{|c|c|c|c|c|}
\hline $\begin{array}{l}\text { Menge des } \\
\text { Autolysats }\end{array}$ & Nicht erwärmt & $\begin{array}{l}3 \text { Stunden } \\
620\end{array}$ & 1 Stunde & $\begin{array}{l}3 \text { Stunden } \\
100^{\circ}\end{array}$ \\
\hline $\begin{array}{l}0,5 \\
0,25 \\
0,1\end{array}$ & $\begin{array}{c}\text { starke Kuppe } \\
\text { do. } \\
\text { do. }\end{array}$ & $\begin{array}{c}\text { incomplet } \\
\text { starke Kuppe } \\
\text { do. }\end{array}$ & $\begin{array}{c}\text { incomplet } \\
\text { starke Kuppe } \\
\text { do. }\end{array}$ & $\begin{array}{c}\text { starke Kuppe } \\
\text { do. } \\
\text { do. }\end{array}$ \\
\hline
\end{tabular}


Ueber die toxischen und hämolytischen Wirkungen der Organantolyse.

b) Autolysat von Rinderleber (20 Tage lang autolysirt.)

\begin{tabular}{c|c|c|c|c}
\hline $\begin{array}{c}\text { Menge des } \\
\text { Autolysats }\end{array}$ & nicht erwärmt & $\begin{array}{c}3 \text { Stunden } \\
62^{0}\end{array}$ & $\begin{array}{c}\text { Stunde } \\
100^{\circ}\end{array}$ & $\begin{array}{c}3 \text { Stunden } \\
100^{\circ}\end{array}$ \\
\hline $\begin{array}{l}0,5 \\
0,25\end{array}$ & $\begin{array}{c}\text { incomplet } \\
\text { starke Kuppe } \\
\text { do. }\end{array}$ & $\begin{array}{c}\text { incomplet } \\
\text { starke Kuppc } \\
\text { do. }\end{array}$ & $\begin{array}{c}\text { starke Kuppe } \\
\text { do. } \\
\text { do. }\end{array}$ & $\begin{array}{c}\text { starke Kuppe } \\
\text { do. } \\
\text { do: }\end{array}$
\end{tabular}

c) Autolysat von Meerschweinchenleber (40 Tage lang autolysirt).

\begin{tabular}{l|c|c|c|c}
0,5 & incomplet & starke Kuppe & Kuppe & Kuppe \\
0,25 & starke Kuppe & do. & starke Kuppe & do. \\
0,1 & do. & do. & do. & do. \\
0,05 & Kuppe & Spur & Spur & Spur
\end{tabular}

d) Autolysat von Mecrsehweinchenniere (37 Tage lang autolysirt).

\begin{tabular}{l|l|l|l|c}
0,5 & 0 & Spur & Kuppe & Kuppe \\
0,25 & 0 & 0 & 0 & 0 \\
0,1 & 0 & 0 & 0 & 0 \\
0,05 & 0 & 0 & 0 & 0
\end{tabular}

Tabelle 3.

Einwirkung erhitzter Organautolysate auf Meerschweinchenblut (1 cem $5 \mathrm{pCt}$.).

e) Autolysat von Rinderleber ( 25 Tage lang autolysirt).

\begin{tabular}{c|c|c|c|c}
\hline $\begin{array}{c}\text { Menge des } \\
\text { Autolysais }\end{array}$ & nicht erwärmt & $\begin{array}{c}\text { Stunden } \\
62^{0}\end{array}$ & $\begin{array}{c}1 \text { Stunde } \\
100^{0}\end{array}$ & $\begin{array}{c}3 \text { Stunden } \\
100^{0}\end{array}$ \\
\hline $\begin{array}{l}0,5 \\
0,25\end{array}$ & $\begin{array}{c}\text { complet } \\
\text { do. }\end{array}$ & $\begin{array}{c}\text { incomplet } \\
\text { do. } \\
\text { incomplet }\end{array}$ & $\begin{array}{c}\text { complet } \\
\text { do. } \\
\text { do. }\end{array}$ & $\begin{array}{c}\text { starke Kupp } \\
\text { do. } \\
\text { do. }\end{array}$ \\
\end{tabular}

f) Autolysat von Rindermilz (37 Tage lang autolysirt).

\begin{tabular}{l|l|l|l|c}
0,5 & 0 & 0 & complet & complet \\
0,25 & 0 & 0 & do. & do. \\
0,1 & 0 & & 0 & do.
\end{tabular}

g) Autolysat von Mecrschweinchenleber (40 Tage lang autolysirt).

\begin{tabular}{l|c|c|c|c}
0,5 & complet & starke Kuppe & starke Kuppe & incomplet \\
0,25 & do. & do. & do. & siarke Kuppe \\
0,1 & incomplet & do. & do. & do. \\
0,05 & Spur & do. & Kuppe & Kuppe
\end{tabular}

h) Autolysat von Meerschweinchennicre.

\begin{tabular}{l|c|c|c|c}
0,5 & Kuppe & starke Kuppe & Kuppe & Kuppe \\
0,25 & do. & do. & do. & do. \\
0,1 & do. & do. & do. & do. \\
0,05 & do. & Kuppe & 0 & 0
\end{tabular}

Diese Versuche zeigen, dass die hämolytische Wirkung von Organautolysat auf Kaninchenblutkörperchen in den mejsten Fällen durch dreistündiges Erwärmen auf 60-620 nur wenig geschädigt oder eher etwas gesteigert wird, und dass das Erhitzen auf $100^{\circ}$ bald Schädigung, bald Stcigerung der hämolytischen Wirkung zu Stande bringt. Das Verhalten crwärmter Organautolysate gegen Mcerschweinchenblut ist auch dasselbe. 
Ob die coctostabile Substanz eine einheitliche Substanz ist, oder ob es sich um ein Gemisch von hämolytisch wirkenden Körpern handelt, können erst weitere Untersuchungen entscheiden.

$\mathrm{Tab}$ e $11 \mathrm{e} 4$.

Rinderleberautolysat auf Kaninchenblut.

\begin{tabular}{|c|c|c|c|}
\hline $\begin{array}{l}\text { Menge des } \\
\text { Autolysats }\end{array}$ & $\begin{array}{c}\text { nicht } \\
\text { behandelt }\end{array}$ & $\begin{array}{l}1 / 50 \text { n. } \mathrm{HCl} \text { zugesetzt, } \\
30 \mathrm{Min} .62^{\circ} \mathrm{C} \text {. erwärmt, } \\
\text { dann neutralisirt }\end{array}$ & $\begin{array}{c}1 / 60 \text { n. } \mathrm{NaOH} \text { zugesetzt, } \\
30 \mathrm{Min} .: 62^{\circ} \mathrm{C} \text {. erwärmt, } \\
\text { dann neutralisirt }\end{array}$ \\
\hline $\begin{array}{l}0,5 \\
0,25 \\
0,1 \\
0,05 \\
0,01\end{array}$ & $\begin{array}{c}\text { starke Kuppe } \\
\text { do. } \\
\text { do. } \\
\text { do. } \\
0\end{array}$ & $\begin{array}{l}0 \\
0 \\
0 \\
0 \\
0\end{array}$ & $\begin{array}{l}0 \\
0 \\
0 \\
0 \\
0\end{array}$ \\
\hline
\end{tabular}

Durch Zusatz stärker alterirender Mittel, Salzsäure und Natronlauge, bei höherer Temperatur wird die hämolytische Wirkung zerstört, wie oben stehende Tabelle zeigt. Bei den oben geschilderten Versuchen wurde immer dafür gesorgt, dass die beim Erwärmen entstandenen Niederschläge vor der Prüfung glcichmässig in der Flüssigkeit vertheilt wurden.

Centrifugirt man den Niederschlag ab, so crhält man einen wirksamen klaren $\Lambda$ theil und dureh $\Lambda$ ufschwemmung des Niederschlages in der entsprechenden Menge physiologischer Kochsalzlösung eine neue Emulsion, die die hämolytische Fähigkeit fast verloren hat, wie dies aus folgenden Versuchen hervorgeht (Tabelle 5).

$$
\text { Tabelle } 5 \text {. }
$$

1. Autolysat von Rinderleber (40 Tage lang autolysirt). Meerschweinchenblut $1 \mathrm{ccm} 5 \mathrm{pCt}$.

\begin{tabular}{|c|c|c|c|c|}
\hline $\begin{array}{l}\text { Menge des } \\
\text { Autolysats }\end{array}$ & $\begin{array}{c}\text { nicht } \\
\text { erwärmt }\end{array}$ & $\begin{array}{c}3 \text { Stunden } \\
62^{\circ}\end{array}$ & $\begin{array}{c}1 \text { Stunde : } 100^{\circ} \\
\text { Niedcrschlag, centri- } \\
\text { fugirt, in Kochsalz- } \\
\text { lösung wieder auf- } \\
\text { geschwemmt }\end{array}$ & $\begin{array}{l}1 \text { Stunde : } 100^{\circ} \\
\text { durch Centrifugiren } \\
\text { gewonnene klare } \\
\text { Flüssigkeit }\end{array}$ \\
\hline $\begin{array}{l}0,5 \\
0,25 \\
0,1\end{array}$ & $\begin{array}{c}\text { complet } \\
\text { do. } \\
\text { incomplet }\end{array}$ & $\begin{array}{c}\text { incomplet } \\
\text { do. } \\
\text { Kuppe }\end{array}$ & $\begin{array}{l}0 \\
0 \\
0\end{array}$ & $\begin{array}{l}\text { incomplet } \\
\text { do. } \\
\text { do. }\end{array}$ \\
\hline
\end{tabular}

2. Autolysat von Rinderleber (40 Tage lang autolysirt). Kaninchenblut $1 \mathrm{cem} 5 \mathrm{pCt}$.

\begin{tabular}{l|c|c|c|c}
0,5 & incomplet & incomplet & 0 & incomplet \\
0,25 & starke Ruppe & starke Kuppe & 0 & do. \\
0,1 & do. & do. & 0 & do.
\end{tabular}

3. Autolysat von Meerschweinchenleber (40 Tage lang autolysirt). Kaninchenblut $1,0 \mathrm{ccm} 5 \mathrm{pCt}$.

\begin{tabular}{l|c|c|c|c}
0,5 & starke Kuppe & starke Kuppe & 0 & starke Kuppe \\
0,25 & do. & do. & 0 & do. \\
0,1 & do. & do. & 0 & do.
\end{tabular}

4. Autolysat von Rindermilz (40 Tage lang autolysirt). Kaninchenblut $1 \mathrm{ccm} 5$ pCt.

\begin{tabular}{l|c|c|c|c}
0,5 & starke Kuppe & starke Kuppe & 0 & Kuppe \\
0,25 & do. & do. & 0 & do. \\
0,1 & do. & do. & 0 & do.
\end{tabular}


Ueber die toxischen und hämolytischen Wirkungen der Organautolysate. 663

Wenn wir das Vorkommen einer hämolytisch wirkenden hitzebeständigen Substanz in den Organautolysaten in gelöster Form annehmen, so ist leicht verständlich, dass dieselbe durch das beim Erhitzen entstandene Coagulum der Flüssigkeit nicht entzogen wird.

Versetzt man 1 Theil eines Autolysats aus Rinderleber mit 10 Theilen 96 proc. Alkohol, filtrirt nach einiger Zeit von dem entstandenen flockigen Niederschlag ab, destillirt das klare Filtrat im Vacuum und nimmt den Rückstand wieder in physiologischer Kochsalzlösung auf, so erhält man eine flockige Suspension von hämolytischer Wirkung. Filtrirt man diese Flüssigkeit, so erweisen sich die vom Filter abgewaschenen Flocken unwirksam, während das klare Filtrat die hämolytische Wirkung entfaltet (Tabelle 6).

$\mathrm{Tab}$ a $11 \mathrm{e} 6$.

Rinderleberautolysat auf Kaninchenblut (1 com 5 pCt.).

\begin{tabular}{c|c|c|c}
\hline $\begin{array}{c}\text { Menge des } \\
\text { Autolysats }\end{array}$ & $\begin{array}{c}\text { Gesammtfüssig- } \\
\text { keit }\end{array}$ & Klares Filtrat & $\begin{array}{c}\text { Aufschwemmung } \\
\text { der Flocken*) }\end{array}$ \\
\hline & complet & complet & 0 \\
1,0 & do. & incomplet & 0 \\
0,5 & incomplet & do. & 0 \\
0,25 & do. & do. & 0 \\
0,1 & Spur & 0 & 0
\end{tabular}

*) Rückstand aus Alkoholdestillat, in 0,85 proc. Kochsalzlösung aufgenommen.

Es ist also klar, dass die bei der beschriebenen Behandlung in der alkoholischen Flüssigkeit gelöste Substanz in Kochsalzlösung auch fast löslich ist.

Ich untersuchte noch in einem Fall die Einwirkung des Autolysats auf Blutkörperchen bei $0^{0}$, um die Möglichkeit einer Trennung eines etwa vorhandenen Amboceptors und Complements festzustellen. Je $1 \mathrm{ccm}$ 5 proc. Meerschweinchenblut wurde in Eis abgekühlt, dann in wechselnden Mengen abgekühltes Rinderleberautolysat zugesetzt und das Gemisch 2 Stunden lang bei. $0^{0}$ gehalten. Hier trat keine Lösung ein. Es wurde dann centrifugirt, das Sediment wieder in Kochsalzlösung $(1,5 \mathrm{ccm})$ aufgenommen und der Abguss mit $0,05 \mathrm{ccm}$ vom Serum befreiten Meerschweinchenblut versetzt (Tabelle 7).

$\mathrm{T}$ a b e 11 e 7 .

Meersehweinchenleberautolysat auf Meerschweinchenblut (1 com 5 pCt.).

\begin{tabular}{c|c|c|c|c}
\hline $\begin{array}{c}\text { Menge des } \\
\text { Autolysats }\end{array}$ & Controle bei 37 & nach $\begin{array}{c}2 \text { Stunden } \\
0^{0}\end{array}$ & $\begin{array}{c}\text { Hämolyse durch } \\
\text { Abguss }\end{array}$ & $\begin{array}{c}\text { Hämolyse durch } \\
\text { Sediment }\end{array}$ \\
\hline & complet & 0 & incomplet & complet \\
1,0 & do. & 0 & do. & do. \\
0,5 & do. & 0 & 0 & do. \\
0,25 & do. & 0 & 0 & do. \\
0,05 & do. & 0 & 0 & incomplet \\
0,01 & incomplet & 0 & & do.
\end{tabular}

Ich unternahm nun noch, die Antikörperbildung zu untersuchen. $\mathrm{Zu}$ peritonealen lnjectionen von Kaninchen brauchte ich das Autolysat 
aus Rinderleber und Meerschweinchenleber. Alle Kaninchen erhielten in gewissen Intervallen achtmal je $15 \mathrm{ccm}$ des Autolysats injicirt und wurden 7 Tage nach der letzten Injection entblutet und die antihämolytische Wirkung gegen Meerschweinchenblut geprüft. Aus diesem Versuche konnte ich keine hemmende Wirkung des Immunserums nachweisen (Tabelle 8). Normales Kaninchenserum entfaltet schon eine hemmende Wirkung auf die Hämolyse dureh Leberautolysat. Diese antihämolytische Wirkung ist nicht auf Antikörper im eigentlichen Sinne zurückzuführen, denn diese Schutzwirkung übersteht den Einfluss höherer Temperatur. Dies stimmt mit dem Verhalten der Schutzwirkung des normalen Kaninchenserums gegenüber der Hämolyse durch Organextract überein.

Tabelle 8 .

1. $1 \mathrm{ccm}$ Meerschwcinchenblut $+0,5 \mathrm{ccm}$ Rinderleberautolysat.

\begin{tabular}{c|c|c|c}
\hline + Immunserum & $\begin{array}{c}\text { Inactivirtes } \\
\text { Immunserum }\end{array}$ & $\begin{array}{c}\text { Inactivirtes } \\
\text { Normal- } \\
\text { kaninchenserum }\end{array}$ & $\begin{array}{c}1 \text { Stunde auf 100" } \\
\text { erhitztes Normal- } \\
\text { kaninchenserum }\end{array}$ \\
\hline 0,2 & 0 & 0 & 0 \\
0,15 & complet & complet & complet \\
0,1 & do. & do. & do. \\
0,05 & do. & do. & do. \\
0,01 & do. & do.
\end{tabular}

2. $1 \mathrm{ccm}$ Meerschweinchenblut $+0,5 \mathrm{ccm}$ Meerschweinchenleberautolysat.

\begin{tabular}{l|c|c|c}
0,5 & Spur & Spur & Spürchen \\
0,15 & complet & complet & complet \\
0,1 & do. & do. & do. \\
0,05 & do. & do. & do. \\
0,01 & do. & do. & do.
\end{tabular}

Die von mir untersuchten hämolytischen Substanzen der Organautolysate sind also von Hämolysinen des Serums ganz verschieden und gehören vielleicht zu einer eigenartigen Gruppe von hämolytisch wirkenden Stoffen.

\section{Resultat.}

1. Die hämolytisch wirkende Substanz ist coctostabil und alkohollöslich und kann ihre Wirkung durch poröse Filter festhalten, im Gegensatz zu den von Morgenroth u. $\Lambda$. untersuchten, hämolytischen Organextracten.

2. Die Substanz ist zur Antikörperauslösung nicht befähigt.

3. Ob diese Substanz schon in der lebenden Zelle als solche präformirt ist, oder ol) sie erst durch den Protoplasmazerfall oder durch den Einfluss der Autolyse entstanden ist, können wir vorläufig noch nicht entscheiden.

\section{Toxische Wirkung des Organantolysats.}

Das Autolysat der Organe wurde ebenso hergestellt, wie oben beschrieben ist. Vor der Anwendung wurde das Autolysat auf $1 / 5$ eingeengt.

Zum Experiment wurde es bei Kaninchen, Meerschweichen, Tauben und Ratten angewendet. Die folgenden Protokolle umfassen nicht die 
gesammte Zahl meiner Thierversuche, sondern geben meistens gleichsam Paradigmata.

\section{A. Versuche mit dem Herzautolysat vom Rinde.}

Vier Meerschweinschen und je zwei Kaninchen, Tauben und Ratten wurde das Autolysat (120 Tage lang autolysirt: Reaction: sehr schwach sauer) intraperitoneal wic subcutan eingespritzt.

Vier Meerschweinchen $(0,3-0,5 \mathrm{ecm}$ pro $\mathrm{kg})$ und ein Kaninchen (3,0 cem : 1000) starben, während Tauben $(2,5-3,0 \mathrm{cem}: 1000)$ und Ratten (5-6 ccm : 1000) gesund blieben. Das zweite Kaninchen wurde nach zwei Injectionen ( $4 \mathrm{ccm}$ im Ganzen, d. h. 2,8 ccm : 1000) getödtet.

Meerschweinchen No. 1 starb nach 2 Tagen. Entzündung an der Injectionsstelle und Anschwellung der benachbarten Lymphdrüsen, Hämorrhagie in der Milz, Niere und Leber. parenchymatöse Degeneration der Niere und leichte partielle Nekrose der Leber, Trübung der Herzmuskelfasern.

Meerschweinchen No. 2. Tod nach 4 Tagen. Alle inneren Organe hyperämisch, Hämorrhagic in den Lungen, Nieren und im Pankreas, parenchymatöse Degeneration der Leber und Trübung der Horzmuskelfasern, intacte Mil $z$.

Meerschweinchen No. 3. Tol nach 4 Tagen. Entzündung an der Injectionsstelle und Anschwollung der danebenlicgenden Lymphdrüsen, keine Veränderung in der Milz, im Magen und Darm, starke Hyperämie in der Leber, in den Lungen, Nieren, Nebennieren und im Pankreas, Hämorrhagien in den Lıngen und Nebennieren, farenchymatöse Degeneration der Niere, Trübung der Herzmuskelfasern.

Meorschweinchen No. 4. Tod nach 3 Tagen. Milz, Leber, Nieren und Lungen hyperämisch, Hämorrhagien in der Milz und den Lungen, hochgradige parenchymatöse Degeneration der Niere und Leber, Trübung der Herzmuskelfasern.

Kaninchen No. 2. Tod nach 10 Tagen. Nieren angeschwollen und Cysten von verschiedener Grösse; die Schnitilächo zeigt deutliche Trübung sowie Harncanälchenerweiterung in der Form von Cysten; Trübung der Leber; Pneumonie.

\section{Resultat.}

Meerschweinchen sind sehr empfänglich dem Rinderherzautolysat gegenüber, während Tauben und Ratten dagegen stark refractär sind, so dass die $\Lambda$ bnahme des Körpergewichts nach 17 Tagen ganz zurücktritt.

Die wichtigsten anatomischen Veränderurigen sind:

1. Entzündung an der Injectionsstelle und Anschwellung danebenliegender J ymphdrüsen.

2. Hyperämie der inneren Organe und Hämorrhagie in denselben.

3. Parenchymatöse Degeneration der Niere und der Jeber.

4. Pneumonie.

5. Trübung der Herzmuskelfasern. 


\section{B. Versuche mit dem Autolysat der Rinderniere.}

Es wurde 3 Kaninchen, 3 Mecrschweinchen, 3 Tauben und 2 Ratten ein Autolysat (120 Tage lang aulolysirt) intraperitoneal eingespritat. Zwei Kaninchen (2 cem : 1000), ein Meerschweinchen (5 cem : 1000) und eine Taube (3 Injectionen, 6,6 ccm im Ganzen, d. h. $19 \mathrm{ccm}: 1000$ ) starben, während zwei Kaninchen $(1,0-1,8 \mathrm{ccm}: 1000)$, ein Mecrschweinchen (4 ccm : 1000), zwei Tauben (6-7,5ccm: 1000) und zwei Ratten $(10-20 \mathrm{ccm}: 1000)$ dauernd gesund blieben.

Meerschweinchen No. 5. Tod nach 27 'Tagen. Hämorrhagien in iler Milz und der Junge, parenchymatöne Degeneration der Niere.

Kaninchen No. 3. Tıd nach 12 Tagen. Eingeweide injicirt, Hämorrhagien in den Jungen.

Kaninchen No. 4. Tod nach 27 Tagen. Slarke Abmagerung, Hämorrhagie in der Linge, Trübung des Nierenparenchyms.

\section{Resultat.}

Kaninchen reagiren empfindlicher als Meerschweinchen.

Die anatomischen Befunde sind:

1. Hyperämie der inneren Organe, ofl auch mit Hämorrhagien.

2. Parenchymalöse Degeneration der Niere.

\section{Versuche mit dem Autolysat der Rindermilz.}

Drei Kaninchen (1,5-2 (em : 1000), drei Meerschweinehen (5 bis 6 ('cm : 1000), zwei Tauben $(8-12 \mathrm{cem}: 1000)$ und zwei Ratten (5 bis $7 \mathrm{ccm}:$ 1000) wurde ein Rindemilzautolysat (120 Tage lang autolysirt, schwach sauer) intraperitoneal eingesprit»t.

lin Meerschweinchen (6 cem: 1000), ein Kaninchen (2 ccm : 1000) und eine Taube $(12 \mathrm{ccm}: 1000)$ starben, während die übrigen Versuchsthiere am Leben blieben. Eine Ratte wurde getödtet.

Kaninchen $\mathrm{N}$ ○. 5. Jod nach 22 Tagen. lingeweide injicirt, sonst nichts Besonderes.

Meerschweinchen No. 7. Tod nach 12 Tagen. Injectionsstelle nekrotisch, dancbenliegende Drüsen geschwollen, Eingeweide hyperämisch, Hämorrhagien jn (ler Leber und subseröse Blutıng im Magen und Dünndarm, hochgradige Trübung der Niere und der Herzmuskelfasern, Milı keine Veränderung.

Ratte No. 4. Nach 20 Tagen getödtet; Mil\% und Niere hyperämisch, Hämorrhagic in den Lungen, parenchymatöse Degeneration der Niere.

Taube No 6. Tod nach 26 Tagen. Milz hämorrhagisch, parenchymaltöse Degeneration der Niere, partielle Nekrose der Leber, Trübung der Herzmuskelfasern.

Resultat.

Die wichligsten Veränderungen sind:

1. Entzündung und Nekrose an der Injectionsstelle.

2. Hyperämie der inneren Organe. 
Ueber die toxischen und hämolytischen Wirkungen der Organautolysate. 667

3. Hämorrhagie in den inneren Organen, besonders in der Lunge.

4. Parenchymatöse Degeneration der Niere und der Leber.

5. Trübung der Herzmuskelfasern.

\section{Versuche mit dem Autolysat der Rinderleber}

(45 Tage lang autolysirt).

Je zwei Kaninchen, Meerschweinchen, Ratten und Tauben wurden verwendet.

Tauben (15-16 cem : 1000), Ratten (10-12 ccm : 1000) and ein Meerschweinchen (4 ccm : 1000) blieben gesund.

Kaninchen No. 9 erhält $6 \mathrm{ccm}$ Autolysat (5 ccm : 1000). Tod nach 10 Tagen. Milz und Niere stark injicirt, Jeber und Niere leicht degenerirt, Trübung der Herzmuskelfasern.

Kaninchen No. 10. Viermalige Injectionen von $10 \mathrm{ccm}$ Autolysat (im Ganzen 7,5 ccm : 1000). 'T'od nach 15 Tagen. Leber, Niere und Lunge hyperämisch, leichte Degeneration des Nierenparenchyms, theilweise Karyorrhexis und Pyknose der Herzmuskelkerne.

Meerschweinchen No. 11 erhält $2,5 \mathrm{ccm} \quad(5,5 \mathrm{ccm}: 1000)$. Tod nach 11 T'agen. Leber, Niere und Lunge stark injicirt, starke Trübung der Leber und der Niere.

\section{Resultat.}

Die Empfindlichkeit der Kaninchen gegenüber dem Autolysat ist etwas grösser als die der Meerschweinchen.

Anatomische Befunde sind:

1. Hyperämie der inneren Organe.

2. Leichte Degeneration der Leber und Nicre.

3. Trübung der Herzmuskelfasern.

\section{E. Versuche mit dem Autolysat von Rinderhoden.}

Es wurde je zwei Kaninchen, Meerschweinchen, Tauben und Ratten ein Autolysat der Rinderhoden (120 Tage lang autolysirt) eingespritzt.

Kaninchen No 7, $1575 \mathrm{~g}$ Gewicht, erhält $30 \mathrm{ccm}$ (27 ccm : 1000) Autolysat. Tod nach 27 Tagen. Sowohl Hyperämie als auch fettige Degeneration der Niere und Leber, im Hoden keine Veränderung.

Kaninchen No. 8, $1010 \mathrm{~g}$ Gewicht, $18 \mathrm{ccm}$ intravenös injicirt. Tod nach 30 Stunden. Innere Organc stark injicirt, Hämorrhagien in der Niere und Lunge, Milz angeschwollen, keine Veränderungen im Eierstock,

Meerschweinchen No. 9, $170 \mathrm{~g}$ Gewicht, erhält $6 \mathrm{ccm}(35 \mathrm{ccm}: 1000)$. Tod nach 5 Tagen. Innere Organe hyperämisch, Milz und Mesenterialdrüsen geschwollen, Hämorrhagien sowohl in der Niere als auch in der Iunge, keine sichtbare Veränderung in den Hoden.

Meerschweinchen No. 11, $170 \mathrm{~g}$ Gewicht, erhält $10 \mathrm{ccm}$. Tod nach 5 Tagen. Hyperämie der inneren Organe, Hämorrhagien in der Niere und Nebenniere, parenchymatöse Degeneration der Niere, Trübung der Leberzellen und der Herzmuskelfasern, keine Veränderungen in den Hoden.

Ratte No. 7, $60 \mathrm{~g}$ Gewicht, erhält zwei subcutane Injectionen (im Ganzen $13 \mathrm{ccm})$. Nach 6 Tagen getödtet. Starke Injeclion sämmtlicher 
Gefässe in der Brust- und Bauchhöhle, Leber trübe, beide Nieren dunkelroth, trübe Schwellung der Rindensubstanz.

Taube No. 18 (15 ccm : 1000) und No. 20 (30 ccm : 1000). Keinerlei Folgeerschcinungen.

\section{Resultat.}

Kaninchen sind auch empfindlich, vertragen aber eine grössere Dosis als gegenüber anderen Autolysaten.

Anatomische Veränderungen sind:

1. Hyperämie nebst Hämorrhagien innerer Organe.

2. Parenchymatöse Degeneration der Niere und Leber.

3. Keine Veränderungen sowohl im Hoden als auch im Eierstock.

\section{F. Versuche mit Leberautolysat von Meerschweinchen.}

Je zwei Kaninchen, Meerschweinchen und Tauben wurden intraperitoneal injicirt.

Ein Kaninchen $(6,6 \mathrm{ccm}: 1000)$ und ein Meerschweinchen (23 cem: 1000 ) ist gestorben.

Kaninchen No. 15. Tod nach 30 Tagen. Starke $\Lambda$ bmagerung, Hyperämie innerer Organe, Hämorrhagien in der Leber, Niere und Lunge.

Meerschweinchen No. 16. Tod nach 11 Tagen. Trübung der Leber, Hämorrhagien in der Niere, Milz hyperämisch, Hämoglobinämie.

\section{Resultat.}

Empfindlicher ist das Kaninchen als das Meerschweinchen.

Anatomische Veränderungen bei Kaninchen sind auch deutlicher.

Die wichtigsten Erscheinungen sind:

1. Hyperämie und Hämorrhagie innerer Organe.

2. Trübung der Leber.

3. Hämoglobinämie.

\section{G. Versuche mit Nierenautolysat von Meerschweinchen.}

Das Autolysat wurde in der Dosis von $3-6 \mathrm{ccm}$ pro $\mathrm{kg}$ zwei Kaninchen intraperitoneal eingespritzt. Das erste Kaninchen starb nach 10 Tagen. Drei Meerschweinchen wurden auch mit der Dosis von 3 bis $8 \mathrm{ccm}$ pro $\mathrm{kg}$ injicirt. Ein Meerschweinchen ist todt.

Kaninchen No. 17 erhält $6 \mathrm{ccm}$ pro kg. Tod nach 10 Tagen. Hyperämie nebst Blutung innerer Organe, leichte Trübung der Leber und Niere.

Meerschweinschen No. 18 erhält $8 \mathrm{ccm}$ pro $\mathrm{kg}$. Tod nach 14 Tagen. Starke Abnahme des Körpergewichts, Hyperämie und Trübung der Leber und Niere, Hämorrhagie in der Lunge, Trübung der Herzmuskelfasern, deutliche Hämoglobinämie.

$$
\text { Resultat. }
$$

Aus diesen Versuchen ersieht man auch, dass das Kaninchen etwas empfindlicher ist als das Meerschweinchen.

Anatomische Veränderungen sind:

1. Hyperämie, Hämorrhagie und Trübung in den inneren Organen.

2. Hämoglobinämie. 
Sämmlliche autolytische Producte zeigten sich in der That toxisch. Das Kaninchen war empfindlicher als das Meerschweinchen, abgesehen von der Wirkung des Herzautolysats. Bei Tauben und Ratten waren grosse Dosen erforderlich, um den Tod herbeizuführen. Versuchsthiere, die tödtlich vergiftet wurden, wurden immer schwächer, konnten sich schliesslich nicht mehr aufrecht erhalten und bisweilen trat unter Streckkrämpfen der Tod ein. Die Excremente waren bisweilen blutig tingirt. Im Harn (der Kaninchen) liess sich Hämoglobin spektroskopisch, oft Eiweiss nachweisen. Bei den Sectionen zeigen sich pathologische Veränderungen hohen Grades an den inneren Organen, und zwar war es im Wesentlichen fast stets dasselbe charakteristische Bild. Vor allem fallen uns die massenhaften, oft eircumscripten Hämorrhagien in den inneren Organen auf und selten auch in den Schleimhäuten und den serösen Häuten.

Ob die Giftigkeit der antolytischen Producte hauptsächlich den während der autolytischen Processe frei werdenden Fermenten von verschiedenen Arten zugeschrieben werden soll, dazu bedürfte es weiterer Untersuchungen, besonders des Thierexperimentes mit den frischen Organfermenten.

Nach Abschluss meiner Untersuchungen an den autolytischen Producten habe ich daher meine Experimente auf die Prüfung einer Toxicität der frischen Organextracte ausgedehnt.

Die Organsäfte wurden in folgender Weise hergestellt: Die dem entbluteten Thiere entnommenen Organe werden mil Quarzsand fein zerrieben, dann mit gleichem Theile physiologischer Kochsalzlösung aufgeschwemmt, einige Stunden bei Zimmertemperatur mehrmals geschüttelt und dann centrifugirt.

\section{Versuche mit Herzsaft vom Rinde.}

Der Herzsaft vom gesunden Rinde wirkt bei Kaninchen in einer Dosis von $19-20 \mathrm{ccm}$ prokg tödtlich. Bei Meerschweinchen $(10-20 \mathrm{ccm}: 1000)$, Taube (6-7 cem : 1000) und Ratte $(35-40 \mathrm{ccm}: 1000)$ treten keine Reactionserscheinungen auf. In Bezug auf das Ergebniss beim Kaninchen war Hyperämie der Leber, der Niere und der Leber, sowie leichte Trübung der Leber und der Harncanälchen nachweisbar.

\section{Versuche mit Nierensaft vom Rinde.}

Frischer Rindernierensaft wurde zwei Kaninchen (3-6 ccm : 1000), zwei Meerschweinchen $(15-20 \mathrm{ccm}: 1000)$ und einer Ratte $20 \mathrm{ccm}: 1000$ ) eingespritzt. Das erste Kaninchen (3 ccm : 1000) starb nach 4 Tagen, während die anderen Versuchsthiere dauernd gesund blieben, indem die Körpergewichtsabnahme allmählich wiederhergestellt war.

Kaninchen No. 14. Hyperämie der Milz, Leber und Niere, hämorrhagische Entzündung der Niere.

\section{Versuche mit Milzsaft vom Rinde.}

Es wurde zwei Kaninchen $(5-6 \mathrm{ccm}: 1000)$, zwei Meerschweinchen (40-45 ccm : 1000), zwei Tauben $(20-25 \mathrm{ccm}: 1000)$ und zwei Ratten 
(40-45 ccm : 1000) Milzsaft vom Rinde intraperitoneal injicirt. Ein Meerschweinchen und eine Ratte gingen unter Abmagerung zu Grunde.

Meerschweinchen No. 23. Tod nach 7 Tagen; Eingeweide hyperämisch, Hämorrhagien in der Lunge, in der Milz keine nennenswerthen Erscheinungen, Nieren- und Leberparenchym leicht degenerirt.

Ratte No. 21. Hyperämie der inneren Organe, Hämorrhagie in der Lunge, der Milz und der Niere sowie im. Herzmuskel.

4. Versuche mit Lebersaft vom Rinde.

Kaninchen No. 22, $3000 \mathrm{~g}$ Gewicht, erhält $15 \mathrm{ccm}$ des Lebersaftes $(5 \mathrm{ccm}: 1000)$. Es waren keinerlei Folgeerscheinungen bemerkbar.

Kaninchen No. 26, $1560 \mathrm{~g}$ Gewicht, erhält $15 \mathrm{ccm}$ (10 ccm : 1000) desselben Saftes. Nach 2 Tagen Tod nach einigen Zuckungen. Die Section ergiebt nur eine mässige Injection der inneren Organe, sonst nichts Besonderes.

Meerschweinchen No. 14, $310 \mathrm{~g}$ Gewicht, erhält $5 \mathrm{ccm}$ (d. h. $17 \mathrm{ccm}$ : 1000). Nach 10 Tagen getödtet. Sectionsbefund: starke Füllung der Mesenterialgefässe, Nieren sehr blutreich, trüb und geschwollen. Zweien Tauben wurden auch derselbe Saft eingespritat (25-30 ccm : 1000). Keinerlei Folgeerscheinungen.

Zwei Ratten erhielten denselben Lebersaft. Die erste Ratte (40 : 1000) starb nach 12 Tagen. Sectionsbefund; Hyperämie der inneren Organe, Trübung der Niere.

\section{Versuche mit Kaninchenlebersaft.}

Er wurde Kaninchen, Meerschweinchen und Tauben intraperitoneal eingespritzt.

Kaninchen No. 17, $2400 \mathrm{~g}$ Körpergewicht, erhält zweimal je $15 \mathrm{ccm}$. Nach 12 Tagen getödtet. Milz und Leber stark injicirt. Trübung der Niere.

Meerschweinchen No. 27, $620 \mathrm{~g}$ Gewicht, erhält $10 \mathrm{ccm}$ vom Lebersaft. Nach 10 Tagen ging das Thier zu Grunde. Befund: Parenchymatöse Degeneration der Rindensubstanz der Niere und Trübung der Leber. Hämorrhagie im Herzmuskel, Pneumonie.

Taube No. 15, $220 \mathrm{~g}$ Gewicht, erhält $9 \mathrm{ccm}$ desselben Saftes. Tod nach 6 Tagen; hochgradige parenchymatöse Degeneration der Nieren, partielle Nekrose der Lieber. Hämorrhagien in der Leber und in der Milz.

6. Versuche mit Lebersaft von Meerschweinchen.

Hier wurde an den Kaninchen, Meerschweinchen, Tauben und Ratten starke Wirkung beobachtet.

Meerschweinchen No. 18, $300 \mathrm{~g}$ Gewicht, erhält $15 \mathrm{ccm}$ (auf zweimal eingespritzt). Tod nach 3 . Tagen; Hyperämie innerer Organe, Hämorrhagie in der Niere und Degeneration der Rindensubstanz derselben, Trübung des Leberparenchyms.

Taube No. 16, $300 \mathrm{~g}$ Gewicht, erhält $12 \mathrm{ccm}$ im Ganzen (auf zweimal eingespritzt). Nach 7 Tagen getödtet; Trübung des Nieren- und Leberparenchyms. 
Ueber die toxischen und hämolytischen Wirkungen der Organautolysate. 671

Ratte No. 12, $230 \mathrm{~g}$ Gewicht, erhält $5 \mathrm{ccm}$. Tod nach 4 Tagen; innere Organe hyperämisch, Hämorrhagie in der Lunge; Leber und Niere trüb.

7. Versuche mit Lebersaft von Tauben.

Kaninchen No. 18, $1000 \mathrm{~g}$ Gewicht, erhält $9 \mathrm{ccm}$. Tod nach 30 Stunden; Hyperämie der inneren Organe, hochgradige parenchymatöse Degeneration der Niere, leichte Trübung der Leber.

Ratte No. 13, $210 \mathrm{~g}$ Gewicht, erhält $4 \mathrm{ccm}$. Tod nach 10 Tagen; starke Füllung der Blutgefässe, Trübung der Leber sowie der Niere.

8. Versuche mit Herzsaft von Kaninchen.

Kaninchen No. 19, $1200 \mathrm{~g}$ Gewicht, erhält $13 \mathrm{ccm}$. Nach 5 Tagen getödtet, keine nennenswerthen Veränderungen.

Meerschweinchen No. 19, $610 \mathrm{~g}$ Gewicht, erhält $8 \mathrm{ccm}$. Tod nach 12 Tagen; Hämorrhagie in der Lunge, Trübung der Niere und Leber, Milz stark injicirt.

Taube No. 17, $330 \mathrm{~g}$ Gewicht, erhält $10 \mathrm{ccm}$ auf zweimal. Nach 3 Tagen getödtet; Hämorrhagie in der Leber, Trübung der Harncanälchen.

Ratte No. 14. $300 \mathrm{~g}$ Gewicht, erhält $3 \mathrm{ccm}$. Nach 12 Tagen getödtet; Hyperämie innerer Organe, Langenentzündung, Trübung der Leber und der Niere.

9. Versuche mit Herzsaft vom Meerschweinchen.

Meerschweinchen No. 20, $490 \mathrm{~g}$ Gewicht, erhält $2 \mathrm{ccm}$. Tod nach 5 Tagen; innere Organe blutreich, Lunge pneumonisch infiltrirt, Hämorrhagie in der Leber sowie in der Niere, Nierenparenchym stark degenerirt.

Kaninchen No. 20, $1400 \mathrm{~g}$ Gewicht, erhält $10 \mathrm{ccm}$. Tod nach 7 Tagen; Trübung des Nierenparenchyms, Milz stark injicirt, Hämorrhagie im Herzmuskelgewebe und in der Lunge.

Taube No. 18, $240 \mathrm{~g}$ Gewicht, erhält $4 \mathrm{ccm}$. Nach 8 Tagen getödtet; Leber blutreich, Nicre trübe und geschwollen.

10. Versuche mit Herzsaft von Tauben.

Taube No. 19, $290 \mathrm{~g}$ Gewicht, erhält $3 \mathrm{ccm}$. Nach 15 Tagen getödtet; sowohl Hyperämie als auch leichte Degeneration der Leber und Niere.

Versuche am Kaninchen (8 ccm : 1000) und an der Ratte $(40 \mathrm{ccm}$ : 1000) auch mit keinerlei Folgeerscheinungen.

11. Versuche mit Nierensaft vom Kaninchen.

Kaninchen No. 21, $2000 \mathrm{~g}$ Gewicht, erhält $19 \mathrm{ccm}$. Nach 15 Tagen getödtet; keine nennenswerthen Veränderungen.

Meerschweinchen No. 21, $220 \mathrm{~g}$ Gewicht, erhält $13 \mathrm{ccm}$. Tod nach 20 Tagen; starke Hyperämie der Milz, Trübung des Nierenparenchyms, Hämorrhagie in der Lunge. 
12. Versuche mit Nierensaft vom Meerschweinchen.

Kaninchen No. 22, $1500 \mathrm{~g}$ Gewicht, erhält $20 \mathrm{ccm}$. Tod nach 21 Tagen; Hyperämie der inneren Organe, stellenweise Rundzelleninfiltration und Harneanälchenerweiterung der Niere.

Meerschweinchen No. 22, $490 \mathrm{~g}$ Gewicht, erhält $5 \mathrm{ccm}$. Tod nach 22 Tagen; Hyperämie der inneren Organe, starke Trübung der Niere sowie der Leber.

13. Versuche mit Nierensaft von der Taube.

Taube No. 20, $290 \mathrm{~g}$ Gewicht, erhält $3 \mathrm{ccm}$. Nach 5 Tagen getödtet; Lungen und Nieren stark injicirt, die letztere auch trübe.

Kaninchen No. 23, $1500 \mathrm{~g}$ Gewicht, erhält $9 \mathrm{ccm}$. Nach 10 Tagen getödtet. Hyperämie innerer Organe; leichte Degeneration der Niere, Trübung der Herzmuskelfasern.

Ratte No. 16, $80 \mathrm{~g}$ Gewicht, erhält $3 \mathrm{cem}$. Tod nach 20 Tagen. Hochgradige Degeneration der Niere, Hämorrhagien in der Lunge und in der Leber.

\section{Résumé.}

Die Giftigkeit der frischen Organextracte war schwächer, als diejenige der autolysirten Producte. Die Krankheitserscheinungen waren aber fast dieselben. Die pathologischen Veränderungen sind qualitativ identisch, aber quantitativ variabel.

Aus obigen Versuchen geht hervor, dass das Autolysat wirksamer ist, als das Extract des betreffenden Organès, das man durch Zerreiben und Centrifugiren erhält. Wenn man die Giftstoffe in dem Autolysat mit denselben in den frisehen Organzellen identificiren könnte, so wäre es wohl wahrscheinlich, dass die Giftstoffe zum grossen Theil durch die Zelltrümmer zurückgehalten seien, die bei der Autolyse vollständig in die Autolysatflüssigeit übergehen und die Giftigkeit des Autolysates steigern.

Vor 4 Jahren hat Muro (18) über thermostabile Gifte in den Organemulsionen geschrieben. Ich habe auch im Obigen die hitzebeständige hämolytische Substanz im Organautolysat schon citirt. Diese interessante Thatsache gab mir Veranlassung, die Wärmebeständigkeit der Autolysatgifte zu prüfen. Ich liess Leberautolysat vom Rinde und Milzautolysat vom Meersehweinchen eine Stunde lang bei $70^{\circ} \mathrm{C}$. und spritzte das erhaltene Autolysat den Thieren - Kaninchen und Meerschweinchen - ein. Die Versuche zeigten keine wesentliche Veränderungen der Giftigkeit im Vergleiche zum Controlgift. Ich will hier nur einige Protokolle anführen.

Kaninchen No. $241800 \mathrm{~g}$ Gewicht, erhält $60 \mathrm{ccm}$ erhitzten Rinderleberautolysats auf 3 Injectionen. Tod nach 32 Tagen: fettige Degeneration der Nieren, Trübung der Leber und Herzmuskelfasern, Hämorrhagie in der J,unge, 
Meerschweinchen No. 26, $280 \mathrm{~g}$ Gewicht, erhält $20 \mathrm{ccm}$ erhitztes Meerschweinchen-Milzautolysat auf 4 Injectionen. 'Tod nach 28 Tagen; Hyperämie innerer Organe, Trübung des Nieren- und Jeberparenchyms, leichte Hämorrhagie in der Niere.

\section{Zusammenfassung.}

Das gesammte Resultat der vorliegenden Untersuchungen lässt sich in Folgendem zusammenfassen:

1. Die autolytischen Producte enthalten eine hämolytische Sub$\operatorname{stanz}$.

2. Die hämolytische Substanz ist coctostabil, alkohollöslich und zur Antikörperbildung nicht befähigt. Sie geht durch poröse Filter.

3. Die autolytischen Producte können die Versuchsthiere krank machen, meistens langsam tödten. hervor.

4. Die locale Wirkung ruft Hyperämie, lintzündung und Nekrose

๖. Die allgemeine Wirkung verursacht Hyperämie und Hämorrhagie in den inneren Organen, Degeneration nnd Nekrose der parenchymatösen Organe.

6. Zerfall der Erythrocyton in vivo ist hier nicht so deutlich, wie durch dic Immunhämolysine.

7. Alle anatomischen Veränderungen sind bei den verschiedenen Organautolysaten qualitativ identisch.

8. Das Autolysatengift ist nicht nur gegen die isogenen Thierarten wirksam, sondern auch gegen die heterogenen. Die dadurch hervorgerufenen Veränderungen der Versuchsthiere sind jedoch analog.

9. Vergiftungserscheinungen durch die normalen Organextracte sind fast analog mit denjenigen durch die Organautolysate, obschon die Wirksamkeit der letzteren noch intensiver als die der ersteren ist.

10. Erhitzte Autolysate entfalten auch Giftigkeit, obwohl sie etwas abgeschwächt ist und die Vergiftungserseheinungen sehr langsam verlaufen.

Zum Schlusse sei es mir gestattet, Herrn Prof. A. Sata für seinen gütigen Rath, Herren Dr. Ischii, Tokunaga und Suzuma für ihre liebenswürdige Unterstützung bei den Untersuchungen meinen besten Dank auszusprechen.

\section{Literatur.}

1. Ueber Autodigestion der Organe. Zeitschr. f. klin. Med. 1891. Bd. 17, Supplement.

2. Ueber die fermentative Eiweissspaltung und Ammoniakbildung in der Leber. Zeitschr. f. physiolog. Chemio. 1900. Bd. 30.

3. Ueber die Autolyse der Lunge. Zeitschr. f. physiolog. Chemie. 1901. Bd. 33.

4. Zeitschr. f. physiolog. Chemie. 1901. Bd. 32.

5. Zeitschr. f. physiolog. Chemie. 1899. Bd. 28.

6. Centralbl. f. Gynäkologie. 1901. 
674 Y. Fukuhara, T'oxische und hämolytische Wirkungen der Organautolysate.

7. Zeitschr. f. physiolog. Chemie. 1901. Bd. 34 .

8. Zeitschr. f. physiolog. Chemie. 1902. Bd. 35.

9. Münchenęr med. Wochenschr. 1902. No. 30.

10. Hofmeister's Beiträge zur chem. Physiolog. 1903. Bd. 3.

11. Ein Beitrag zur Chemie maligner Geschwülste. Zeitschr. f. physiolog. Chemie. 1899. Bd. 27.

12. Annales de l'Institut Pasteur. 1899.

13. Centralbl. f. Bakteriologie. 1901. Bd. 30.

14. Wiener klin. Wochenschr. 1901.

15. Annales de l'Institut Pasteur. 1902.

16. Berliner klin. Wochenschr. 1902. No. 37.

17. Osaka Igakkai-Zassi. 1905. Bd. 4. No. 10.

18. Chugai Ijishimpo. 1904. No. 586. 